\title{
Du bon usage de la vodka
}

How to Make Good Use of Vodka

О ПРАВИЛЬНОМ ИСПОЛЬЗОВАНИИ ВОДКИ

\section{Philippe Mennecier}

\section{CpenEdition}

Journals

Édition électronique

URL : http://journals.openedition.org/ethnoecologie/6223

DOI : $10.4000 /$ ethnoecologie.6223

ISSN : 2267-2419

\section{Éditeur}

Laboratoire Eco-anthropologie et Ethnobiologie

Référence électronique

Philippe Mennecier, «Du bon usage de la vodka », Revue d'ethnoécologie [En ligne], 18 | 2020, mis en ligne le 31 décembre 2020, consulté le 29 janvier 2021. URL : http://journals.openedition.org/ ethnoecologie/6223; DOI : https://doi.org/10.4000/ethnoecologie.6223

Ce document a été généré automatiquement le 29 janvier 2021.

\section{(c) (i) $९$}

Revue d'ethnoécologie est mis à disposition selon les termes de la licence Creative Commons Attribution - Pas d'Utilisation Commerciale - Pas de Modification 4.0 International. 


\title{
Du bon usage de la vodka
}

\author{
How to Make Good Use of Vodka \\ О ПРАВИЛЬНОМ ИСПОЛЬЗОВАНИИ ВОДКИ
}

Philippe Mennecier

Boire sa vodka tout seul,

Ce serait merveilleux!

Mais c'est toujours à deux qu'on fume, Mais c'est toujours à trois qu'on lampe.

Et pour toi seul que reste-t-il?

Le berceau et le tombeau ${ }^{1}$.

Vladimir Vyssotski

1 En Europe, il y a une culture du vin et une culture de la vodka, qui se distinguent pour des raisons climatiques évidentes. Mais, si la viticulture s'accompagne d'une différenciation des terroirs et des goûts, la production de vodka n'est guère différenciée, même s'il existe un grand nombre de marques.

\section{L'origine de la vodka}

2 Le mot «vodka » est un diminutif, propre au parler slave de Russie, formé sur le mot voda, « eau », et signifie donc littéralement " petite eau ». Le terme apparaît au Moyen Âge comme une façon de désigner l'eau. Il prend peu à peu le sens d'eau-de-vie à partir du XIV ${ }^{\mathrm{e}}$ siècle et, jusqu'au XIX ${ }^{\mathrm{e}}$, il n'a cette signification que dans les régions céréalières des gouvernements de Moscou, Koursk, Orlov, Tambov, et dans la région de Kharkov au nord-est de l'Ukraine (Pohlëbkin 2009).

3 En Russie, la vodka devient la boisson nationale au XVIII ${ }^{\mathrm{e}}$ siècle, par un privilège accordé à la noblesse en 1716. Toutefois, il ne s'agit pas encore de la vodka que l'on connaît aujourd'hui. Le dictionnaire de Vladimir Dahl, le «Littré russe ", paru en 1863-1866, définit encore le mot vodka comme un distillat de vin, de grain ou de fruit (Dahl 1863-1866). 
4 En réalité, le vin n'a jamais été un breuvage propre à la Russie. Il était importé de Byzance et d'Asie centrale. Dans l'empire russe, on en produisait dans les régions plus clémentes du Caucase, de Crimée et de Bessarabie. Des vins du Rhin et de Bourgogne furent également importés dès le $\mathrm{x}^{\mathrm{e}}$ siècle dans la Russie de Novgorod.

5 La vodka actuelle, à base d'alcool rectifié de froment ou de pomme de terre, ne date que des années 80-90 du XIx lorsque le ministre des finances, le comte Sergueï Witte, instaura le monopole d'État sous couvert de lutte contre l'alcoolisme et de préservation de la santé des sujets de l'empire (Rodionov 2011). C'est en 1895 qu'apparaît la vodka actuelle à 40 degrés, à base de $40 \%$ d'alcool rectifié à 96 degrés minimum pour $60 \%$ d'eau. La sagesse populaire prétend que ce volume de $40 \%$ était la proportion idéale préconisée par Dmitri Mendeleïev, qui n'y était cependant pour rien, mais qui s'était intéressé aux « Composés d'alcool et d'eau ».

6 Au début de la Première Guerre mondiale, le gouvernement tsariste met en place la prohibition, qui sera maintenue après la révolution d'Octobre, durant la guerre civile, jusqu'en 1925. Apparaît alors une vodka plus douce, à 30 degrés, surnommée facétieusement «rykovka», du nom du président du Conseil des commissaires du peuple, Alekseï Rykov.

7 En 1941, sous Staline, la vodka à 40 degrés est rétablie dans les normes nationales standard, qui codifient également des vodkas à 50 et 56 degrés. Ces nouvelles normes voient le jour après le décret du 26 juin 1940 sur «le passage à la journée de huit heures, à la semaine de sept jours et l'interdiction pour les ouvriers et les employés de quitter sans autorisation les entreprises et les établissements ». Autrement dit, il s'agit de noyer dans la vodka toute forme de résistance à l'esclavage institutionnalisé sous prétexte de l'effort de guerre.

8 Avec la mise en place de la "restructuration ", dite perestroïka, en 1985, Gorbatchev cherche à combattre l'alcoolisme et démantèle de nombreuses usines. Puis, en 1992, Eltsine, plus intempérant et donc plus tolérant, supprime le monopole d'État. La pénurie entraîne la production clandestine d'alcool frelaté qui fait des ravages dans la population. En 1993, le monopole doit donc être rétabli.

\section{La culture de la vodka}

9 Comme le dit un écrivain russe, « la vodka est assurément le premier des breuvages. [...] Car elle est absolument rationnelle. La vodka est faite pour résoudre un seul et unique problème : comment s'enivrer (à quel point, c'est votre problème). Tous les autres breuvages inventés par l'humanité se cachent honteusement derrière une feuille de vigne de qualités gustatives. Ne nous leurrons pas, la vodka n'a pas de goût. Une bonne vodka, c'est la moins mauvaise. Ce sont les zakouskis qui lui donnent son goût » (Makarevič 2005).

10 Et c'est bien ce qui fait la différence fondamentale entre la culture de la vodka et la culture du vin, de la bière ou des spiritueux : ici, on boit pour le goût et la dégustation partagée, là, on boit exclusivement pour le résultat et l'ivresse socialisée.

11 D'ailleurs, le vocabulaire lié à la consommation de vodka, qui est incommensurable, ne porte nullement, comme chez nous, sur la variété des arômes et des saveurs, ou sur les terroirs, mais sur les processus et les résultats. Il existe au moins une centaine de verbes pour suppléer à la froide neutralité du mot "boire», parmi lesquels 
ostogrammit'sja, «se prendre cent grammes", litt. «se cent-grammer», et l'intraduisible opohmelit'sja, "prendre un verre de vodka pour se dessoûler de la cuite de la veille».

12 La littérature soviétique en rend bien compte et notamment, bien entendu, le chefd'œuvre de Venedikt Erofeev (1973), Moskva-Petuški, où l'odyssée alcoolisée du narrateur revisite allègrement la phraséologie bureaucratique.

13 En règle générale, cependant, les Soviétiques ne consommaient pas de boissons alcoolisées en dehors des fêtes civiles ou familiales. Au quotidien, on accompagnait le repas de thé, de kéfir, de kompot ou de mors².

14 En Russie, lors des festins, l'art de la table n'est pas fondamentalement différent du nôtre, mais l'ivresse n'est pas, comme en Europe occidentale, un effet secondaire de la dégustation de vins et d'alcools divers au cours du repas. Le but est de procurer et maintenir une légère ivresse par une absorption de vodka progressive et synchronisée. De là tout un rituel, qui s'est particulièrement affiné durant la période soviétique où la vodka jouait un rôle social unificateur et égalitaire: la tablée constituait une sorte d'espace de socialisme réel où la liberté de parole était admise car incontrôlable.

Par conséquent, les convives ne sirotent pas leur verre quand bon leur semble, à la manière des individualistes œnophiles. La consommation est égalitaire, rythmée et contrôlée par les toasts successifs.

Le repas ne commence pas avant le premier toast prononcé par le maître de maison et le premier verre - en général, un petit verre à liqueur, rjumka - vidé d'un trait ${ }^{3}$ et immédiatement adouci par une zakuska $a^{4}$. On ne déguste pas la vodka, on l'avale comme un remède. Les zakouskis, improprement traduits par amuse-gueule, sont fondamentaux dans la culture de la vodka. En effet, puisque la vodka idéale n'a aucun goût, c'est l'accompagnement qui fournit tout le plaisir gustatif. Il y a des débats enflammés sur les meilleurs zakouskis qui n'ont rien à envier à nos débats sur les avantages du bordeaux et du bourgogne. C'était en Union soviétique l'un des thèmes de discussion préférés dans les taxis, tout de suite après le passage obligé sur le plus grand poète russe : Pouchkine ou Lermontov? Evtouchenko ou Voznessenski? Il y a toutefois un consensus sur les poissons fumés ou marinés (harengs, sprats, etc.), le lard gras (onctueux mais ferme), le caviar sur du pain de seigle de Borodino à la coriandre, une cuiller de borchtch, c'est-à-dire tout ce qui peut faire passer la brûlure de l'alcool. Mais à défaut, un concombre aigre-doux ou un champignon mariné feront l'affaire, au pire... une pincée de sel sur la «tabatière » de la main. Cela dit, certains puristes considèrent que le premier toast ne doit pas s'accompagner de zakuska.

\section{La redistribution égalitaire}

17 «Entre le premier et le second, l'intervalle est faible ${ }^{5}$ », est-il convenu de dire pour proposer le second toast, qui permet à l'un des convives de remercier pour l'hospitalité de la maison. C'est alors, « entre le second et le troisième qu'on atteint le sommet de l'harmonie avec le monde, et, si l'humanité trouvait le moyen de pérenniser cet état, la question du bonheur éternel serait résolue. Hélas, après le second vient toujours le troisième, ce qui est très bien mais déjà moins divin, et l'on passe alors aux conversations profondes, qui s'approfondissent encore avec les toasts suivants» (Makarevič 2016). 
Cependant, le troisième toast ne se fait guère attendre non plus, appelé rituellement par la formule « Dieu aime la Trinité " », autrement dit « jamais deux sans trois ».

Le quatrième et le cinquième toasts peuvent encore être introduits par une formule rituelle, plus variable et moins nécessaire : «La maison a quatre coins » ou « La télègue a quatre roues ", "Dieu n'est pas bête, il aime les pièces de cinq ${ }^{7}$ " (cette dernière formule étant un octosyllabe à rime interne : Bog ne durak - ljubit pjatak).

Au delà, la comptabilité se perd jusqu'au dernier verre, dit « pour le bâton ${ }^{8}$ » [de pèlerin ou de chemineau], l'équivalent plus plébéien du « coup de l'étrier ».

On peut penser que si seuls les cinq premiers toasts et le dernier sont nommés, c'est qu'ils constituent la structure même d'une griserie bien contrôlée, propre à l'intelligentsia raffinée. Au-delà de cette norme civilisée, on passe aisément de la griserie à l'ébriété. Il est d'ailleurs troublant de voir que deux écrivains « dissidents de l'intérieur ", Andreï Bitov (1989) et Venedikt Erofeïev (1976) ${ }^{9}$ ont comparé, pratiquement en même temps et sans doute sans se concerter, la gradation éthylique des levers de coude à un voyage en train et à la succession des stations sur les voies de chemin de fer ${ }^{10}$.

La répartition des toasts est rythmée par le maître de maison qui doit veiller que personne ne s'enivre exagérément, ce qui dérogerait aux règles de la bienséance. Le principe est que chacun parvienne à une légère ivresse partagée et contrôlée, tempérée par l'absorption des aliments, à cet état de bien-être parfois appelé le kaiif ${ }^{11}$.

3 Le maître de cérémonie peut également être un tamada, choisi par les convives, coutume empruntée à la Géorgie, où l'art du toast est légendaire. Celui-ci " rafraîchit ${ }^{12}$ " les verres avant chaque toast, c'est-à-dire qu'il y complète la quantité de vodka, personne n'étant vraiment tenu de faire cul sec à chaque fois. En effet, le verre doit être empli à ras bord, afin que « la vie soit bien remplie».

Notons qu'une bouteille entamée (un litre ou un demi-litre) doit toujours être entièrement vidée, et on ne peut qu'admirer l'art avec lequel l'amphitryon sait répartir équitablement un fond de bouteille entre tous les convives. Le même principe vital évoqué pour le verre interdit la présence d'une bouteille vide sur la table.

Cette consommation socialiste de la vodka s'est probablement institutionnalisée au cours de la période soviétique. Les tablées improvisées étaient l'une des principales distractions, qui permettaient de s'abstraire de la morosité et de la défiance générales. Les convives ne répondaient pas forcément à une invitation, il arrivait fréquemment que les amis s'invitent à l'improviste, souvent sans même téléphoner, mais sans oublier la quantité de vodka et de zakouskis nécessaires.

Une fois l'ivresse égalitaire atteinte, toute parole non conforme avait l'excuse de l'ivresse partagée et pouvait être réfutée. Aussi une personne sobre n'était-elle pas la bienvenue, comme témoin éventuel d'une parole dissidente, voire comme un délateur potentiel. Quand des convives arrivaient au cours du repas, on leur faisait boire immédiatement autant de verres « d'amende ${ }^{13}$ » qu'on en avait bu jusqu'alors afin de les hisser au niveau d'ivresse acquis. Depuis la perestroïka, cette sage coutume redistributive semble être tombée en désuétude.

Comme les tablées étaient souvent improvisées au dernier moment, il fallait se procurer de la vodka à une heure où la vente était interdite (Erofeev 1973:25) ${ }^{14}$. Les soiffards pouvaient toujours se fournir dans les arrière-cours auprès de revendeurs qui 
leur glissaient les bouteilles par la fenêtre. Et les moins fortunés ne manquaient pas de revendre les bouteilles vides dans les « points de consigne » officiels ${ }^{15}$.

\section{Le principe de solidarité}

De même que la consommation modérée, conviviale, la consommation excessive, stakhanoviste, s'adapte aux conditions environnementales.

Dehors, par moins 30 degrés, prendre cent grammes de vodka ${ }^{16}$ procure une voluptueuse mais perfide sensation de chaleur et de bien-être sans ivresse qui permet d'affronter une longue marche dans le froid ou un travail en extérieur. "Vous imaginez une soirée d'hiver en Sibérie avec des pelmenis ${ }^{17}$ et une bouteille de chardonnay?» (Makarevič 2005).

Durant la période soviétique, la paie était versée en liquide le $1^{\mathrm{er}}$ et le $15 \mathrm{du}$ mois. Après avoir rapporté l'argent à la maison, les gens ne gardaient que peu d'argent en poche. Les buveurs avaient donc coutume de se cotiser à trois pour acheter et boire sur place une bouteille de $50 \mathrm{cl}$, qui valait environ trois roubles. Cela s'appelait « combiner à trois ${ }^{18} »$ : pour recruter deux comparses, l'initiateur se postait debout à contre-courant $\mathrm{du}$ flot des passants en glissant sa main dans sa veste et en agitant les trois doigts du milieu. La question rituelle était: "Tu seras le second?», puis "Tu seras le troisième ${ }^{19}$ ? ».

31 Pour se réchauffer en hiver par grand froid, on peut aussi prendre 50 grammes de spirt, c'est-à-dire de la vodka à 98 degrés. Il est alors conseillé de boire d'abord un verre d'huile ou de crème fraîche pour éviter les brûlures d'œsophage et d'expirer avant et après l'absorption pour préserver les poumons d'effluves délétères. L'effet de chaleur est immédiat, mais l'ivresse ne s'installe brusquement que si l'on rentre dans une pièce chauffée.

C'est tout le danger de la consommation de la vodka en hiver : les ivrognes peuvent se coucher dans la neige et s'endormir définitivement. Mais il y a en Russie, et pour cause, une grande compassion pour les victimes de l'alcool. À l'époque soviétique, les passants venaient au secours des buveurs endormis dans le froid ou appelaient la milice, qui les emmenait au "dessoûloir ${ }^{20}$ ». Cette solidarité était quasi institutionnelle en Russie soviétique, où la moindre trace d'alcool au volant était passible d'une lourde peine de prison : en vous rendant à une soirée, vous pouviez convenir avec le chauffeur de taxi de venir vous rechercher dans quelque état que vous fussiez, de vous ramener chez vous et de vous installer dans votre lit.

33 Le mode de consommation de la vodka apparait bien comme un reflet d'une civilisation où la distance spatiale entre les individus, chère à Edward T. Hall (1971), est certainement plus rapprochée qu'en Europe occidentale, où le contact physique est plus sensible, tantôt brutal, tantôt chaleureux, une civilisation de la compassion et de la sociabilité, attachante et parfois étouffante, et qui laisse peu de place à l'individualisme et au besoin de solitude.

Les Russes n'ont généralement pas conscience de cette spécificité de leur mode de consommation de la vodka qui peut d'ailleurs les amener à boire cul sec les meilleurs vins et alcools étrangers, car, jusqu'à une date récente, ces boissons ne faisaient pas vraiment partie de leur culture. 
(a) claire comme de l'eau de roche, sans robe, sans arômes, sans terroir, n'est pas faite pour être dégustée. C'est la dévoyer que de la consommer avec d'autres spiritueux ou d'en faire une base de cocktail. Autre chose est d'en faire un rehaut nécessaire à la consommation de bière, comme le souligne une autre formule consacrée : «Bière sans vodka, c'est de l'argent à tous les vents ${ }^{21} »$. un strict rituel collectif ${ }^{22}$.

\section{BIBLIOGRAPHIE}

Bitov A. 1989 - La Maison Pouchkine, traduit par Philippe Mennecier. Paris, Albin Michel.

Dahl V. (ВЛАДИМИР ДАЛЬ) 1863-1866 - ТОЛКОВЫЙ СЛОВАРЬ ЖИВОГО РУССКОГО ЯЗЫКА [Dictionnaire raisonné de la langue russe vivante], ИЗДАНИЕ ОБЩЕСТВА ЛЮБИТЕЛЕЙ РОССИЙСКОЙ СЛОВЕСНОСТИ, УЧРЕЖДЕНОГО ПРИ ИМПЕРАТОРСКОМ МОСКОВСКОМ УНИВЕРСИТЕТЕ, МОСКВА.

Erofeev V. (ВЕНЕДИКТ ЕРОФЕЕВ) 1973 - МОСКВА - ПЕТУШКИ, ИЗД. ҮMСА Press, ПАРИЖ.

Erofeiev V. 1976 - Moscou-sur-Vodka, traduit par Annie Sabatier et Antoine Pingaud. Paris, Albin Michel,.

Hall E.T. 1971 - La dimension cachée, traduit par Amélie Petita. Paris, Le Seuil.

Makarevič А. (АНДРЕЙ МАКАРЕВИЧ) 2005 - ЗАНИМАТЕЛЬНАЯ НАРКОЛОГИЯ [Narcologie amusante], ИЗД. МАХАОН.

Makarevič А. (АНДРЕЙ МАКАРЕВИЧ) 2016 - « Pro vodku », www.argolis-yacht.ru/Articles/ Makarevich_Pro-vodku.htm

Pohlëbkin V. V. (ВИльям ВАСИЛЬЕВИч ПОХЛЕБКИН) 2009 - ИСтоРИя вОДКИ [Нistoire de la vodka]. ИЗДАТЕЛЬСТВО ЦЕНТРПОЛИГРАФ.

Rodionov В. (БОРИС РОДИОНОВ) 2011 - ИСТОРИЯ РУССКОЙ ВОДКИ ОТ ПОЛУГАРА ДО НАШИХ ДНЕЙ [L'histoire de la vodka russe depuis le polougar jusqu'à nos jours], эксмо, М.

\section{NOTES}

1. ЕСЛИ Б ВОДКА БЫЛА НА ОДНОГО - / КАК ЧУДЕСНО БЫ БЫЛО! / НО ВСЕГДА ПОКУРИТЬ - НА ДВОИХ, / НО ВСЕГДА РАСПИВАТЬ - НА ТРОИХ. / ЧТО ЖЕ - НА ОДНОГО? / НА ОДНОГО - КОЛЫБЕЛЬ И МОГИЛА.

2. Kompot : décoction froide de fruits entiers ; mors : décoction de pulpe de fruits mélangée au jus frais.

3. En principe, on choque systématiquement les verres à la fin de chaque toast, sauf s'il est porté à la mémoire d'un défunt.

4. Zakuska (pl. zakuski) du verbe zakusit', litt. «accompagner qch. en mordant (kusat')», par exemple, [Oblonski] zakusil vodku rybkoj, litt. «Oblonski accompagna sa vodka avec du poisson » (Tolstoï, Anna Karénine).

5. МЕЖДУ ПЕРВОЙ И ВТОРОЙ ПРОМЕЖУТОК/ПЕРЕРЫВЧИК НЕБОЛЬШОЙ. 
6. БоГ ТРОИцУ люБит. L'expression joue sur le double sens du mot troica : « Trinité » ou « triade ».

7. У ДОМА ЧЕТЫРЕ УГЛА ; У ТЕЛЕГИ ЧЕТЫРЕ КОЛЕСА ; БОГ НЕ ДУРАК - ЛЮБИТ ПЯТАК.

8. НА ПОСОШОК.

9. Les ouvrages cités ont été écrits respectivement entre 1964 et 1971 et en 1969, bien avant d'être sortis clandestinement d'Union Soviétique et édités à l'étranger.

10. «Ainsi battait le pouls du temps et haletait l'espace jalonné par les arrêts-vodka. Tantôt à deux, tantôt à trois, tantôt à dix, puis de nouveau à cinq, ils célébraient une bouteille, toujours la même, semblait-il. La chose la plus inexorable, la chronologie, variait très affablement après chacune de ces doses contradictoires, et la postériorité cessait de défiler bien sagement derrière l'antériorité, toutes deux étaient sorties des rangs et s'étaient mises à marcher d'un pas léger comme si le temps avait décidé de passer en tête à tête avec lui-même cette aimable soirée où le présent attendait le passé et où le futur était arrivé avant tout le monde. » (Bitov 1989 : 333).

11. Kaïf (КАЙ $)$, emprunt à l'arabe, 1821 : « état de délectation, d'ivresse, euphorie ». Pojmat', polučit' kajf (ПоЙмАТЬ, ПоЛУчИТЬ КАЙФ), « atteindre le kaïf ».

12. ОСВЕЖИТЬ : « rafraîchir ».

13. ШТРАФНЫЕ РЮМКИ : « verres d'amende ».

14. «Ô période bénie dans la vie de mon peuple, de l'ouverture à la fermeture des magasins ! » (O, БЛАЖЕННЕЙШЕЕ ВРЕМЯ В ЖИЗНИ МОЕГО НАРОДА - ВРЕМЯ ОТ ОТКРЫТИЯ И ДО ЗАКРЫТИЯ МАГАЗИНОВ!).

15. ПУНКТЫ ПРИЁМА СТЕКЛОТАРЫ.

16. Au détail, la vodka se vend au poids, par multiples de 50 grammes.

17. Pelmenis (ПЕльмЕни), plat sibérien (le mot vient en réalité d'une langue finno-ougrienne de l'Oural), sortes de raviolis, généralement farcis de viande de porc et de mouton.

18. СООБРАЗИТЬ НА ТРОИХ.

19. ВТОРОЙ / ТРЕТИЙ БУДЕШЬ?

20. ВЫТРЕЗВИТЕЛЬ.

21. ПИВО БЕЗ ВОДКИ - ДЕНЬГИ НА ВЕТЕР.

22. Je remercie Serge Bahuchet qui m'a suggéré d'écrire cet article lors d'une discussion autour d'une bouteille de vodka. Je remercie également Boris Chichlo, Oleg Fedorenko, Irina Moltchanova et Bielka Nemirovski pour leur relecture attentive et leurs suggestions judicieuses.

\section{RÉSUMÉS}

La culture de la vodka est très différente de celle du vin. La vodka ne se différenciant pas par des terroirs et n'ayant aucune saveur particulière, on ne la boit pas pour son goût, mais pour ses effets. Un rituel presque immuable permet de partager et de maintenir une agréable griserie collective. La succession codifiée des toasts, appelés par des formules consacrées, renforce la cohésion des convives.

Vodka culture is very different from that of wine. Vodka does not differ according to the regions, and it has not any particular flavour: they don't drink it for the pleasure of its taste, but for its effects. An almost immutable ritual offers partaking and maintaining a pleasant collective inebriation. The codified succession of the toasts, called by accepted formulas, strengthen guest' cohesion. 
КУЛЬТУРА ПИТИЯ ВОДКИ РЕЗКО ОТЛИЧАЕТСЯ ОТ КУЛЬТУРЫ ПИТИЯ ВИНА. ТАК КАК ВОДКА ОДНА И ТА ЖЕ ВО ВСЕХ УГОЛКАХ СТРАНЫ И НЕ ИМЕЕТ ОСОБОГО ВКУСА, ЕЕ И ПОТРЕБЛЯЮТ НЕ РАДИ ВКУСА, А РАДИ ЭФФЕКТА. В РОССИИ СУЩЕСТВУЕТ ПОЧТИ НЕИЗМЕННЫЙ РИТУАЛ ДЛЯ РАЗДЕЛЕНИЯ УДОВОЛЬСТВИЯ КОЛЛЕКТИВНОГО ПОТРЕБЛЕНИЯ ЭТОГО НАПИТКА И ПОДДЕРЖАНИЯ ПРИЯТНОГО ОПЬЯНЕНИЯ. ЧЕТКО ОПРЕДЕЛЕННОЕ ЧЕРЕДОВАНИЕ ТОСТОВ, СОПРОВОЖДАЕМЫХ СООТВЕТСТВУЮЩИМИ СЛУЧАЮ ПОГОВОРКАМИ, УКРЕПЛЯЕТ СПЛОЧЕННОСТЬ СОТРАПЕЗНИКОВ.

INDEX

motsclesru РОССИЯ, ВОДКА, ЗАКУСКИ, ТОСТЫ, РИТУАЛ, ЛЕКСИКА, ПОГОВОРКИ

Keywords : Russia, vodka, zakuski, toasts, ritual, lexicon, sayings

Mots-clés : Russie, vodka, zakouskis, toasts, rituel, lexique, dictons

\section{AUTEUR}

\section{PHILIPPE MENNECIER}

Attaché honoraire Muséum national d'Histoire naturelle, UMR 7206 Eco-anthropologie 